\title{
Trapped positron flux formation in the innermost magnetosphere of the Earth
}

\author{
A. A. Gusev ${ }^{1}$, U. B. Jayanthi ${ }^{1}$, G. I. Pugacheva ${ }^{1}$, V. M. Pankov² , and N. Schuch ${ }^{3}$ \\ ${ }^{1}$ Instituto Nacional de Pesquisas Espaciais, INPE, Sao Jose dos Campos, SP, Brazil \\ ${ }^{2}$ Space Research Institute of Russian Academy of Science, Moscow, Russia \\ ${ }^{3}$ Southern Regional Space Research Center/INPE, Santa Maria, Brazil
}

(Received December 5, 2001; Revised April 6, 2002; Accepted April 20, 2002)

\begin{abstract}
The possible existence of a positron radiation belt in the inner magnetosphere of the Earth, its space location, flux, energy distribution and the ratio of $e^{+} / e^{-}$fluxes is considered. The source of the positrons/electrons is assumed to be the decay of charged pions $(\pi-\mu-e$ decay chain) produced, in the nuclear interactions between protons and the neutral constituents of the atmosphere. The production of excess positron fluxes over electron ones through this process is examined for two different proton populations and their atmospheric interactions in the altitude range of 80 to $1000 \mathrm{~km}$. Monte Carlo simulations of intra-nuclear cascade process through SHIELD code was utilized to simulate these interactions. Considering the trapped proton fluxes in the inner magnetosphere as a source, the simulations for interactions in rarified atmosphere shows excess of positrons over electrons with ratios above 2 for proton energies below $2.5 \mathrm{GeV}$. Although, protons above this energy do not produce excess of positrons compared to electrons in atmospheric interactions, we assumed the primary cosmic rays with the energy greater than 8-10 $\mathrm{GeV}$ also as a source for these interactions, and utilizing the east-west asymmetry in the arrival directions of these primary cosmic rays combined with the exponential nature of the atmosphere density we also obtained an excess of positrons over electrons escaping from the atmosphere to the altitudes of satellite orbits. A comparison is attempted with the recent data of AMS experiment on board the space shuttle.
\end{abstract}

\section{Introduction}

Nuclear interactions of cosmic ray protons with the Earth's atmosphere are a well-known source of charged and neutral energetic particles in the magnetosphere. These interactions take place in the innermost region of the magnetosphere where the atmosphere has sufficient density to produce a noticeable number of secondaries but low enough to permit these product particles to escape from the atmosphere. The strength of the geomagnetic field in this region is practically the same as that at the Earth's surface and the influence of this relatively strong magnetic field on the secondary charge particle fluxes produces several significant geophysical effects. The most noticeable of them is the energetic $(\geq 10 \mathrm{MeV})$ proton radiation belt created through the decay of energetic secondary neutrons escaping from the atmosphere.

The secondary charged particles produced in the atmosphere are registered at satellite altitudes as albedo; splash, re-entrant or quasi-trapped particles. The dependence of the primary cosmic ray flux on the cut-off rigidity results in an increase of the intensity of the secondaries (both charged and neutrals) with increasing latitude. As the cosmic ray cut-off rigidity depends on the angle of incidence with respect to the geomagnetic east direction, this azimuthal dependence of the primary cosmic ray flux results in azimuthal dependence of secondary fluxes.

Copy right (c) The Society of Geomagnetism and Earth, Planetary and Space Sciences (SGEPSS); The Seismological Society of Japan; The Volcanological Society of Japan; The Geodetic Society of Japan; The Japanese Society for Planetary Sciences.
The same as the primary cosmic rays, the trapped energetic protons in the radiation belt also interact with the residual atmosphere and generate further secondaries. These trapped secondary particles can constitute new components of the radiation belt, including, for example, the isotope radiation belts. However, the species and spectra of the fluxes of the secondary radiation produced by the primary cosmic rays and the trapped proton radiation differ. Hereafter, to distinguish between the origins of these secondaries, the cosmic ray source and trapped proton sources are referred below to as $\mathrm{CR}$ and TP respectively.

Freden and White (1960) were the first to point out the possibility that energetic TP can generate light element isotope ions like $\mathrm{D}, \mathrm{T},{ }^{3} \mathrm{He}$ which could be geomagnetically trapped. This hypothesis of isotope radiation belt was confirmed experimentally by the observations of CRRES and SAMPEX satellites (Chen et al., 1994; Selesnik and Mewaldt, 1996; Spjeldvik et al., 1998a, b). Earlier, Basilova et al. (1982) considered the energetic TP as a source of tens to hundreds $\mathrm{MeV}$ trapped positrons and electrons in the inner zone and this is a valid hypothesis to explain their observations and the results of Galper et al. (1983) and Voronov et al. (1987).

The simulations made to obtain the absolute fluxes of secondary trapped positrons and electrons with rigidities less than $\approx 8 \mathrm{GV} / \mathrm{c}$, the minimal cut-off rigidity in the equatorial region, are presented here. As we are considering relatively lower-energetic particles, these do not escape from the magnetosphere and can perform total or partial longitudinal drift around the Earth. In our earlier estimates of the 
trapped positron and electron fluxes of this nature (Gusev et al., 1996, 2001), we utilized essentially approximate and averaged parameters for the nuclear reaction kinematics (energy and the angular distributions of secondary particles are averaged and approximated). Here, we utilized more precise energy and angular distributions for the secondary products of the nuclear reactions of cosmic rays and trapped protons with the atoms of the residual atmosphere.

\section{Positron Flux from the Trapped Proton Source}

Both positrons and electrons from nuclear reactions of energetic protons with the nuclei of the residual atmosphere are presumed to be produced through the chain of decays of pions and muons:

$$
\begin{aligned}
& \pi^{ \pm} \rightarrow \mu^{ \pm}+v ; \quad \mu^{ \pm} \rightarrow e^{ \pm}+v+\tilde{v} \\
& \pi^{0} \rightarrow 2 \gamma \rightarrow 2 e^{+}+2 e^{-}
\end{aligned}
$$

The necessary minimum energy of incident proton for $\pi$ meson production is $\approx 290 \mathrm{MeV}$.

Due to short lifetime of pions and muons the electrons are generated very close to the point of nuclear interaction. The electrons and the positrons produced in the nuclear interactions through muon decays are thus essentially confined to the same $L$-shells of the parent energetic trapped protons. As the maximum in intensity of these protons is located at $L \approx 1.2$, the trapped secondary positrons also are concentrated within this region. In contrast the $\gamma$-quanta from the decay of $\pi^{0}$ travel a long distance from the point of generation, and not being affected by the geomagnetic field, escape from the magnetosphere or sink into more denser atmosphere and do not contribute to the electron flux produced by TP.

To simulate a nuclear reaction yield, we initially computed the pion production spectra (originated in $1 \mathrm{~g} / \mathrm{cm}^{2}$ of the atmospheric matter) using a well-tested version of the Monte Carlo computer code SHIELD for the intra-nuclear cascade simulations (Dementyev and Sobolevsky, 1999). As helium is the main constituent of the Earth's atmosphere at altitudes of $\sim 800 \mathrm{~km}$ (the minimum altitude of geomagnetic equator of $L$-shell 1.2), the simulation was performed with helium target atoms although the pion production has a weak dependence on the target atomic number A. We calculated the distributions of pion output with respect to energy and angle in the laboratory system. The energy distributions of muons were obtained by normalizing to the pion energy by a coefficient of 0.8 , which corresponds to the average part of the energy carried away by muon in $\pi-\mu$ decay. In the muon rest system, the angular distribution of the decay electrons is isotropic and their energy spectrum is normalized as described by $d N e / d E_{e}=E^{2}\left(3.0-4.0 \mathrm{E} / \mathrm{mc}^{2}\right) / 74198.4$ (Barashenkov and Toneev, 1972). Through relativistic transformations from the muon rest system to the laboratory system and considering the energy and angle of each muon, the distributions were transformed to calculate the positron/electron production spectra.

In Fig. 1, we show the positron excess over electrons as obtained by the computations of the positron/electron production spectra generated by protons of various energies in nuclear interactions with atmospheric helium (Gusev et al., 2001). For incident protons at energies in the range of 0.3 to

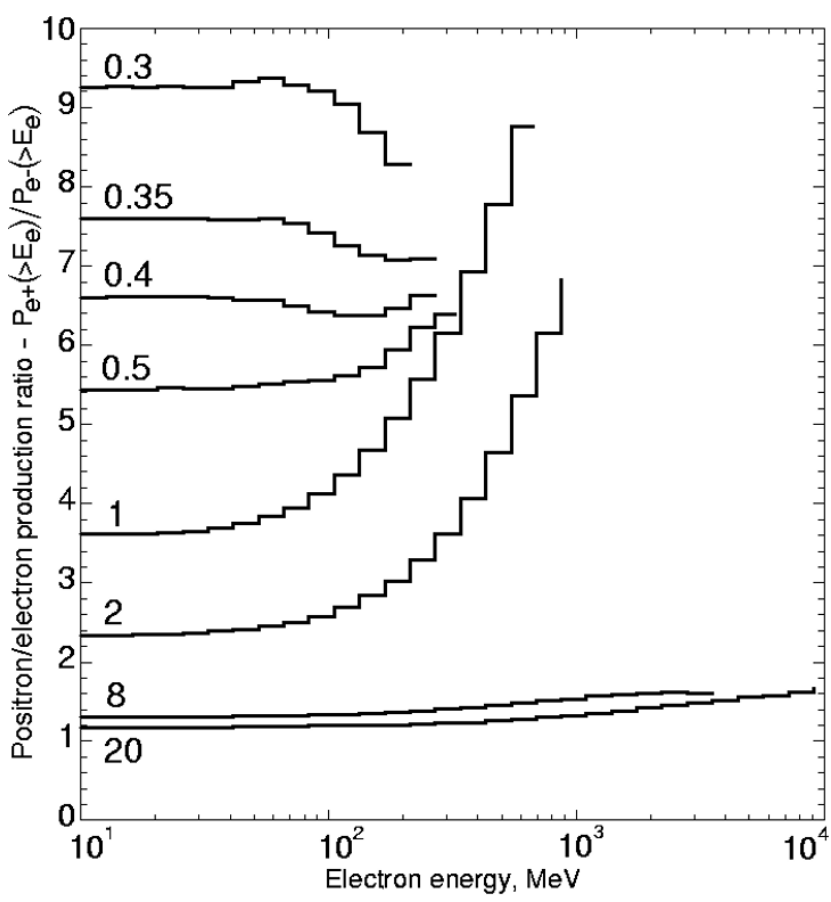

Fig. 1. The dependence of positron/electron production ratio $P_{e^{+}}\left(>E_{e}\right) /$ $P_{e^{-}}\left(>E_{e}\right)$ on electron energy for helium target. Numbers near the curves mark an incident parent proton energy in $\mathrm{GeV}$.

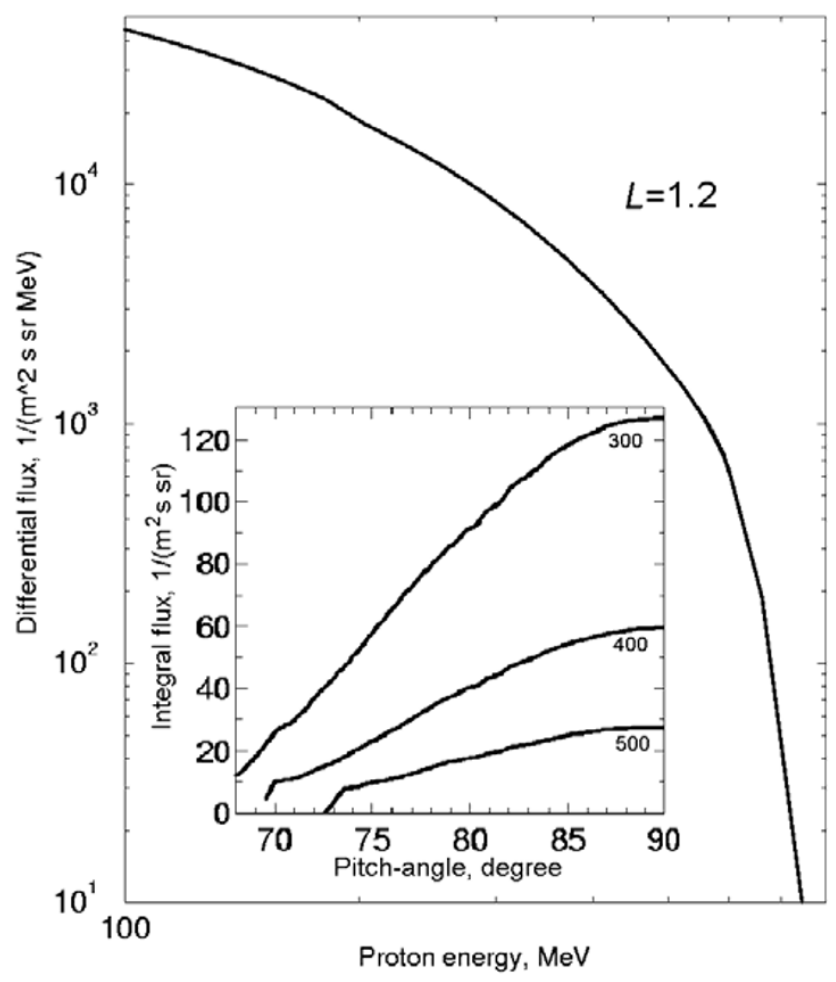

Fig. 2. The spectrum and the geomagnetic equatorial pitch-angle distribution of the trapped proton fluxes at $L$-shell of 1.2 according to AP8-min model. The numbers next to the curves mark proton energies.

$2.0 \mathrm{GeV}$, and for electrons with energies of about $10 \mathrm{MeV}$, the integral production fluxes ratios of pions are in general $>2$ and reach a maximum value of $\approx 9$ near $300 \mathrm{MeV}$, the energy corresponding to the reaction energy threshold. For 


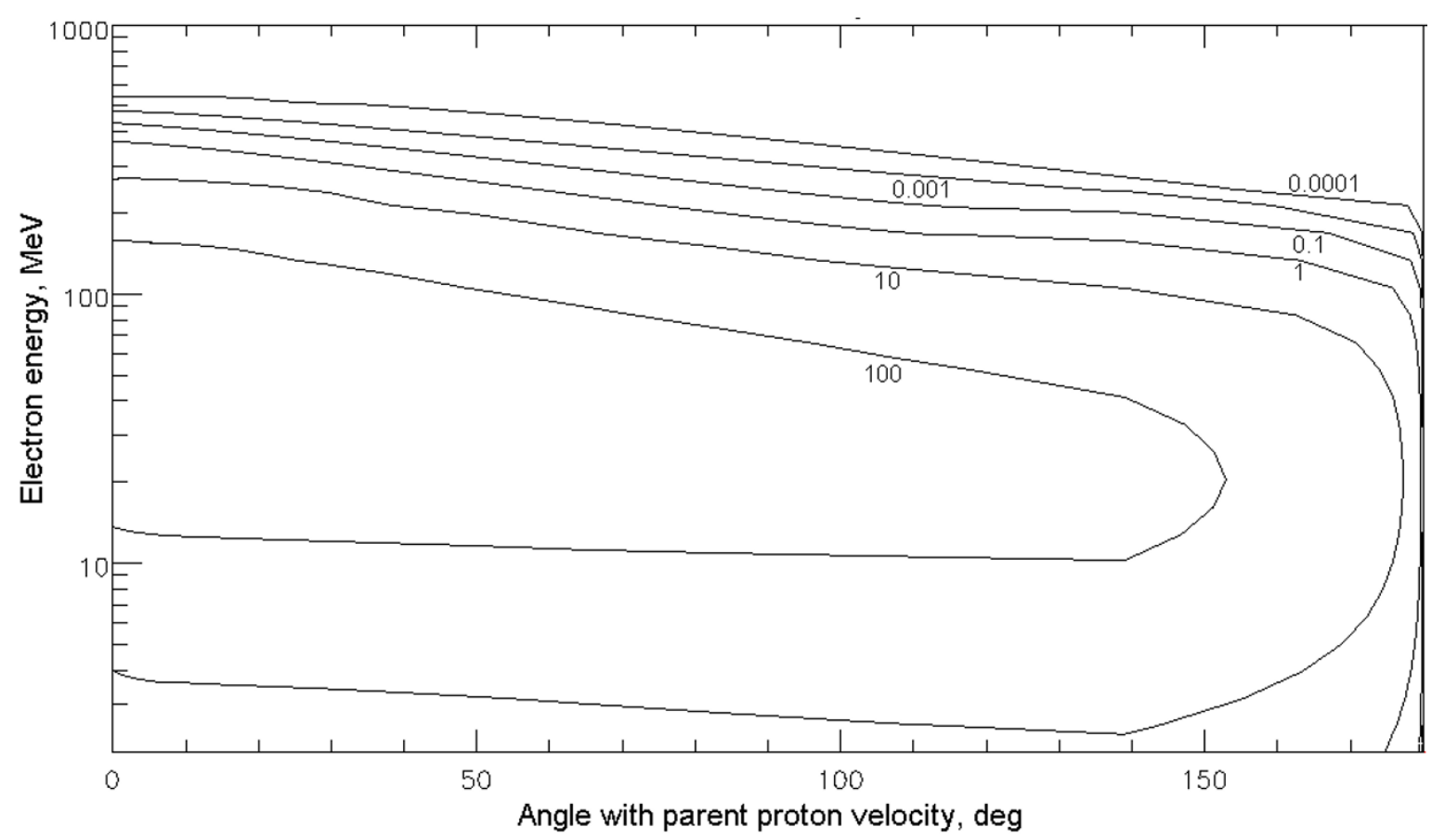

Fig. 3. The angle-energy distribution of positrons produced in $\pi \rightarrow \mu \rightarrow e$ decays. Numbers near the curves indicate the differential positron production flux in units of positrons/MeV s sr g.

protons of energies of $\sim 0.5$ to $2.0 \mathrm{GeV}$, this ratio increases to values of about 10 with increasing positron energy. Qualitatively, this result is a direct consequence of the charge conservation law which tends to favor positive pion production when only a few pions are born in the nuclear reactions $p+A \rightarrow D+\pi^{+}+A^{\prime} ; p+\pi^{+}+A^{\prime}$, and $p+n+\pi^{+}+A^{\prime}$ predominating near the reaction threshold energy (Machner and Hidenbauer, 1999), where $A^{\prime}$ is a target atomic number of exited nucleus after reaction.

Thus, nuclear reactions of the trapped proton component with $0.3-2.0 \mathrm{GeV}$ energy substantially favor the production of positrons over electrons in the energy range of about 10 $\mathrm{MeV}$ to several hundreds $\mathrm{MeV}$. This important feature is quite unlike the situation for the CR protons in the geomagnetic equatorial region. These protons with minimal energy of $\approx 8 \mathrm{GeV}$, produce a greater number of pions and consequently the ratio of positron to electrons is almost equal to unity.

Because the trapped proton component has significantly greater intensity in comparison with the CR protons, at least at $L$-shell of 1.2 where a maximum intensity of energetic trapped protons is located, and especially near the reaction threshold energy, the TP source could predominate over the CR source, and provide enhanced trapped positron fluxes in comparison to electrons. The characteristics of the TP proton source extracted from AP8 solar minimum model (AP8, 1996) for $L$-shell of 1.2 are shown in Fig. 2. The energy spectrum is very steep and indicates a break at energies of $\approx 800 \mathrm{MeV}$ and the angular distribution of the flux is within approximately $18^{\circ}$ around the geomagnetic equatorial plane.

In Fig. 3, we show an example of differential production spectra $P_{e^{+}}\left(E_{e}^{\prime}\right)$ of positrons generated by unidirectional TP flux at $L=1.2$. Although the resulting angular distribution of production fluxes is nearly isotropic, only those positrons, which have the same pitch angles as the trapped protons will be captured by the magnetic field. An estimate of the solid angle of confinement at $L=1.2$ is equal to $1.4 \pi \mathrm{sr}$.

To calculate the positron/electron differential flux $F_{e^{ \pm}}\left(E_{e}\right)$ we must integrate the differential production spectrum $P_{e^{ \pm}}\left(E_{e}^{\prime}\right)$ along the positron trajectory considering that a positron of energy $E_{e}$ was born with energy $E_{e}^{\prime}$ at a distance of $X^{\prime} \mathrm{g} / \mathrm{cm}^{2}$ from the point of observation. Then the positron flux of energy $E_{e}$ at the point of observation is determined by the particle conservation law, which can be expressed as:

$$
\begin{aligned}
& F_{e}\left(E_{e}\right) \Delta E \\
& \quad=\int_{0}^{\infty} P_{e}\left(E_{e}^{\prime}, X^{\prime}\right) \Delta E^{\prime}\left(E_{e}, \Delta E, E_{e}^{\prime}\left(X^{\prime}\right)\right) d X^{\prime},
\end{aligned}
$$

where $\Delta E^{\prime}\left(E_{e}, \Delta E, E_{e}^{\prime}\left(X^{\prime}\right)\right)$ is the function which represents the variation of the energy interval $\Delta E$, with changing energy along the positron trajectory. As shown by Pugacheva et al. (1997), if the production spectrum $P_{e}$ and the positron/electron energy losses $(d E / d x)$ do not depend on $X^{\prime}$ (i.e. the both depend only on $E_{e}^{\prime}$ ) then the integral (1) reduces to

$$
F_{e}\left(E_{e}\right)=\frac{P_{e}\left(>E_{e}\right)}{\left(\frac{d E}{d x}\right)_{E_{e}}}
$$

where $P_{e}\left(>E_{e}\right)$ is an integral positron/electron production spectrum.

The total energy losses of energetic positrons trapped in the inner radiation zone are given by Pugacheva et al. (1997):

$$
\begin{array}{r}
\frac{d E}{d x}=0.002+0.028 E+3.86 \cdot 10^{-6} E^{2} B^{2} / c \bar{\rho} \\
\mathrm{GeV} /\left(\mathrm{g} / \mathrm{cm}^{2}\right), \quad E \text { in } \mathrm{GeV}
\end{array}
$$




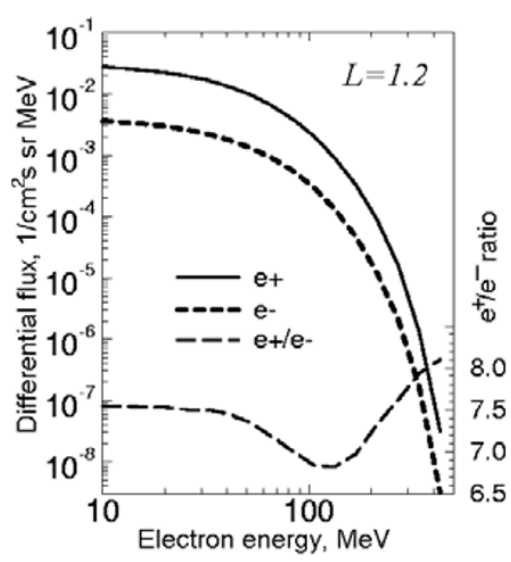

Fig. 4. Spectra of the trapped positrons and electrons produced by trapped proton source at $L=1.2$.

Here the first and second terms represent the ionization and bremsstrahlung losses and the third term is the synchrotron radiation energy losses (with $\bar{\rho}$ as the mean atmospheric density along the electron drift trajectory around the Earth). The synchrotron radiation losses are negligible only in the region very close to the Earth. At the magnetic equator along the field line $L=1.2$ where $B=0.312 L^{-3}=$ 0.18 Gauss and $\bar{\rho} \approx 10^{-17} \mathrm{~g} / \mathrm{cm}^{2}$, for electrons with energies $>100 \mathrm{MeV}$ synchrotron radiation energy losses surpass the ionization and bremsstrahlung losses: $(d E / d x)_{\text {synchr }} \geq$ $(d E / d x)_{\text {ion+brem. }}$.

The results of the simulations are presented in Fig. 4. The characteristic feature of this TP source for trapped positrons is the high $e^{+} / e^{-}$output ratio approximately $7-8$, for the positrons with energies of 10-500 MeV.

\section{Trapped Positron Flux from the Cosmic Ray Source}

CR protons also produce positrons and electrons in nuclear reactions with the residual atmosphere. These secondaries can be trapped at the same $L$-shells where TP generate positron radiation belt. Below we consider the pion production by the $\mathrm{CR}$ protons only. The CR helium spectrum with energies more than 8-10 GeV/n, produces approximately $19 \%$ pions as the spectrum contains $19 \%$ of hadrons in relation to the CR proton spectrum (Guinzburg, 1984).

It is natural to expect that the trapped positron fluxes from CR source could be less intensive due to the low intensity of CR fluxes at the same $L$-shells, as for example, at $L=1.2$ where they are 5-6 orders of magnitude less in comparison with TP flux. However, the multiplicity of pions produced by $\mathrm{CR}$ protons of tens of $\mathrm{GeV}$ is greater than that due to 300 $\mathrm{MeV}$ TP component. As the pion production spectrum depends on both the source flux intensity and the multiplicity, it is not so obvious if absolute values of trapped positron fluxes generated by TP exceed those produced by CR at the same $L$-shells.

Considering the CR source at $L$-shells $1.09,1.12$ and 1.2, and taking into account the $\mathrm{CR}$ arrival directions, the estimates of the trapped positron fluxes and the $e^{+} / e^{-}$flux ratio were made in this modeling (Pugacheva et al., 2001). Figure 5 shows the proton CR spectra composed on the base of

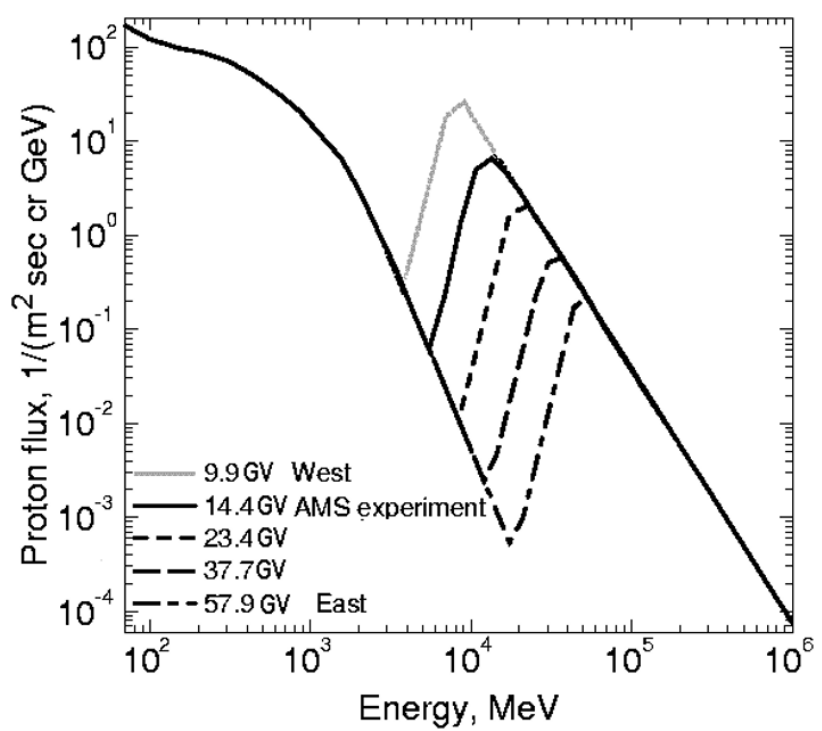

Fig. 5. The parent proton spectra for the geomagnetic equator region at $L=1.09$ in dependence on arrival direction. The spectra consist of the primary and secondary components (The AMS collaboration, 2000a).

the results of The AMS collaboration (2000a) used for the simulation of secondary positrons/electron fluxes. The CR spectra at low latitudes noticeably depend on CR arrival direction, i.e. on the angle of their velocities with the eastern direction resulting in the so-called azimuthal asymmetry in the CR flux. The spectra in Fig. 5 correspond to different cut-off rigidities determined by arrival direction of CR flux. The spectrum sums both the secondary and primary components. At energies below $3 \mathrm{GeV}$ the spectrum also includes secondaries, which are independent of rigidity as they are produced locally. The primary component decreases with rigidity and the flux has the relation $\approx 7000 /\left(R_{\text {cutoff }}\right)^{3 / 2}$. As a result the CR protons arriving from the western direction have about 14 times higher intensity compared to those arriving from the eastern direction.

Figure 6 demonstrates an angle-energy distribution of the positrons/electrons generated by the CR protons. This distribution has a noticeable anisotropy for particles with energies $\geq 40 \mathrm{MeV}$ and has a maximum in the direction of the parent proton. This permits simplification in the calculations by assuming that all the secondary positrons move in the same direction as the parent protons of CR. As it is shown beneath this anisotropy is one of the reasons that positron trapped flux produced by CR exceeds the electron one.

The positrons and electrons are mostly produced in nuclear reactions at the lowest atmospheric altitudes corresponding to selected $L$-shell. The minimal altitude $\left(H_{\min }\right)$ for an $L$-shell of 1.09 is $\approx 80 \mathrm{~km}$, which approximately corresponds to the atmospheric boundary, i.e. it is the minimal closed $L$-shell where charged particles could be captured by the magnetic field and at which they can make at least one drift rotation around the Earth. The corresponding $H_{\min }$ values for $L=1.12$ and $L=1.2$ shells are at $250 \mathrm{~km}$ and $800 \mathrm{~km}$ respectively. Positrons and electrons with pitch angles of about $90^{\circ}$ drift along the geomagnetic equator around the Earth in the opposite directions due to radial gradient of the geomagnetic field. Figure 7 shows how the positrons and 


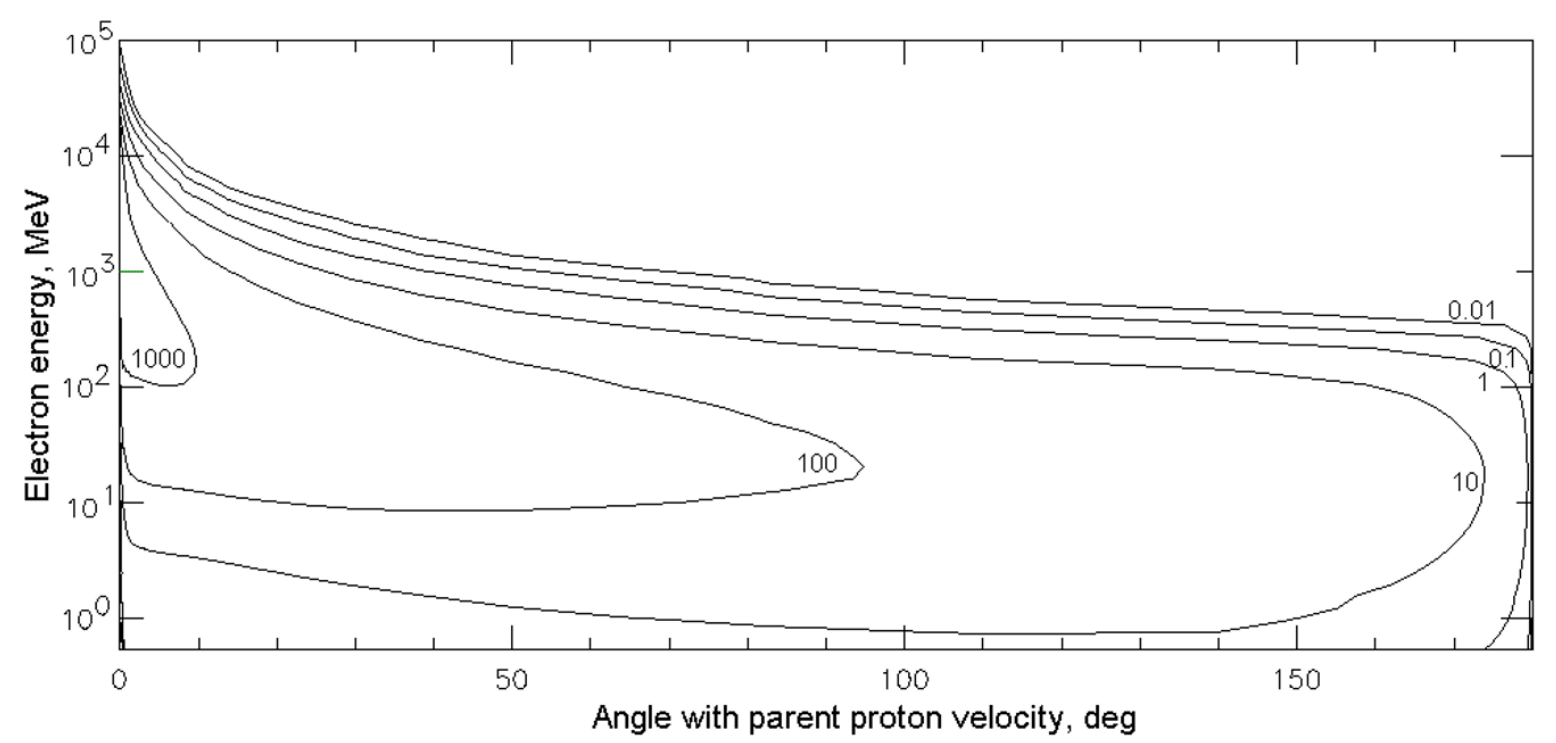

Fig. 6. The angle-energy distribution of positrons like in Fig. 3 but produced by CR.

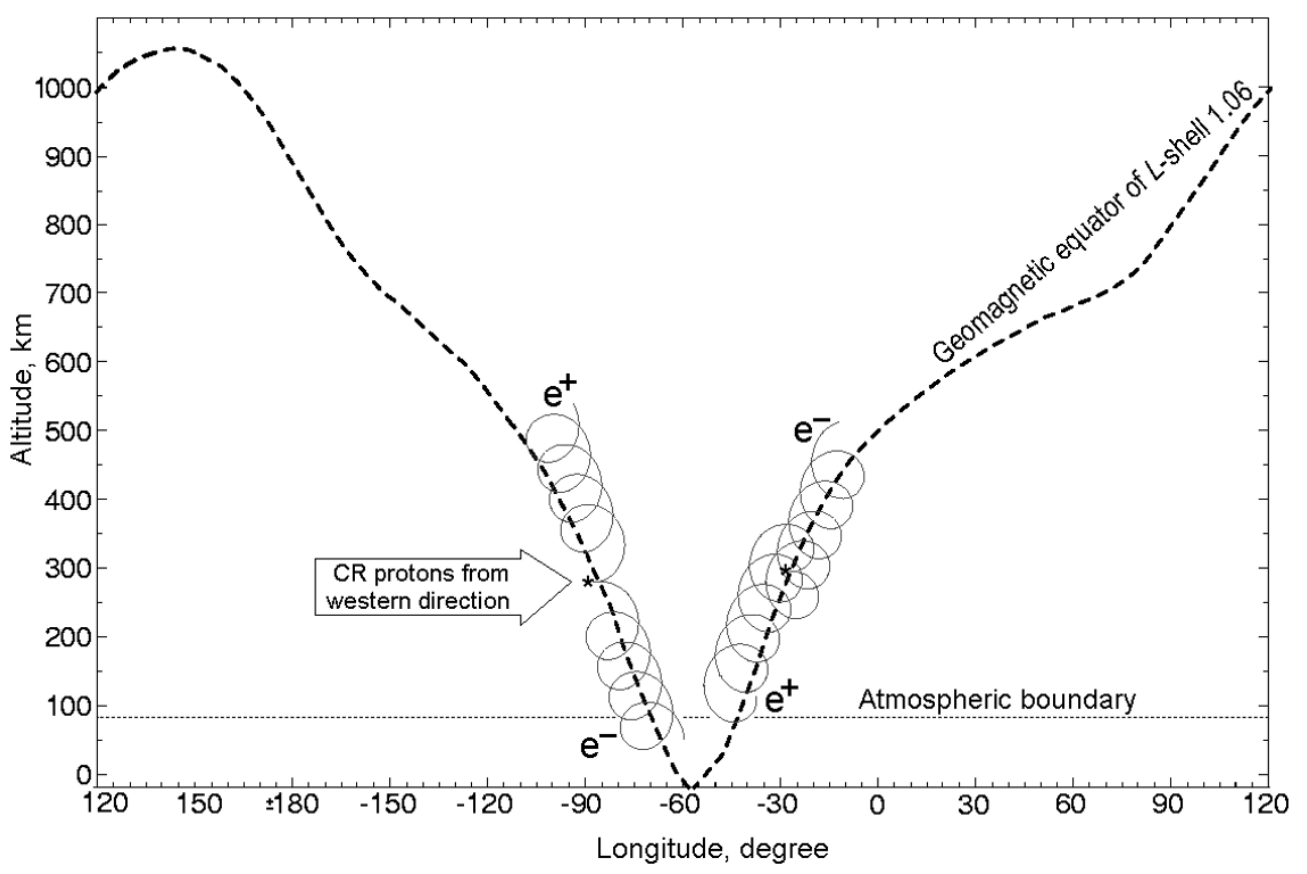

Fig. 7. Drift of the positron and electron generated by western protons along the geomagnetic equator at along unclosed $L=1.06$. Energy losses in the atmosphere are not taken into account in the figure.

electrons produced by the western CR protons (which has the maximal intensity) drift along the geomagnetic equator line of $L=1.06$ (broken line) from the production point. The upward moving particles can escape from the atmosphere and reach satellite altitudes when the downward moving particles sink into the atmosphere and are lost.

In Fig. 8 the simulated trajectories of generated positron and an electron starting from the atmospheric boundary are sketched. The upward drifting electrons produced by CR from western arrival direction have their guiding center beneath the production point and the positrons have the guiding centers above. Due to that the positrons pass through the more rarified atmosphere and escape from the lower lay- ers of the atmosphere unlike the electrons, which encounter denser atmosphere and are lost there. The higher the electron energy, the deeper it sinks into the atmosphere and more rapidly it is lost. In the same time the CR protons coming from the opposite eastern direction, produce particles, which behave differently i.e. only the electrons, escape from the atmosphere. As the intensity of CR protons from eastern arrival directions is lower compared to the western CR component, the escaping electron flux is significantly lower compared to those of positrons. This mechanism favors excess of escape of positrons to higher altitudes compared to electrons. And more over, the greater the difference between the densities at the top and at the bottom of the Larmor cir- 


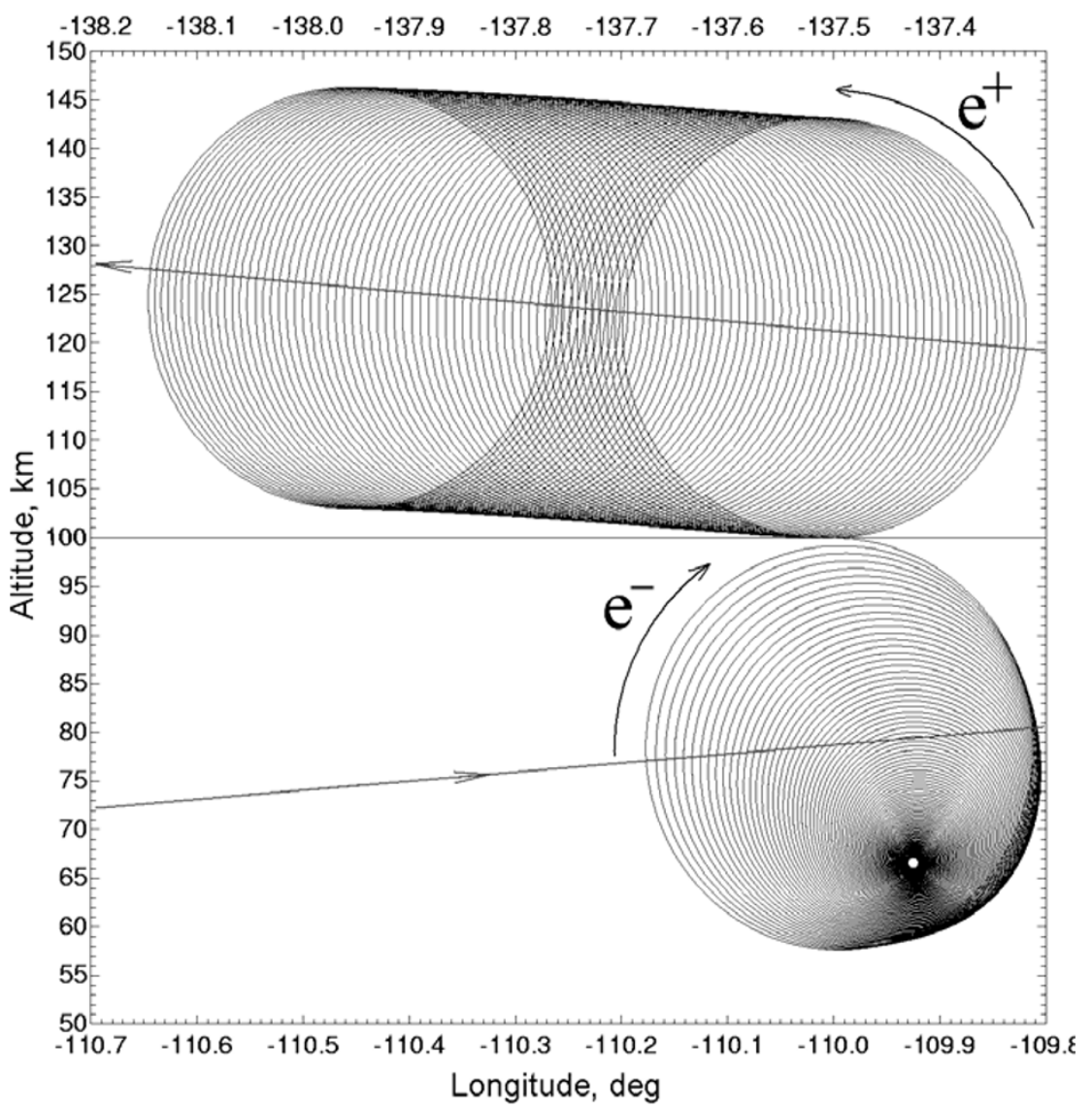

Fig. 8. Simulated trajectories of a positron (upper curve) and an electron (lower curve) born at the same point at the altitude of $100 \mathrm{~km}$, drifting upward along the geomagnetic equator with the guiding centers above (for positrons) and beneath (for electrons) the point of generation. The curved arrows show a direction of Larmor rotation. The straight lines with the arrows show the geomagnetic equator line and magnetic drift direction.

cle, the greater is this difference between the positron and electron fluxes. In the intermediate case of vertical incident CR protons, both the electrons and positrons escape from the atmosphere with the same losses.

In the absolute flux simulations, as the production spectrum changes along the particle trajectory, due to the dependence of the cut-off rigidity of the parent CR protons on arrival directions, we utilized Eq. (1) and not the (2). The numerical integration of Eq. (1) is performed over the quantity of the matter that a particle encounters along the trajectory. The maximal input to the integral (1) comes from the lower part of the Larmor circle where the denser atmosphere favors more interactions.

Figure 9 demonstrates the results of the simulations of trapped positron and electron fluxes at $L=1.09$ generated by the CR component and for comparison the data of AMS experiment is also shown. Also is shown in Fig. 9, the positron/electron fluxes modeled with Eq. (2) where an azimuthal asymmetry of CR is not considered. In this simulation we utilized the vertical CR spectrum observed by AMS at $L<1.09$. In the last case there is no excess of positron fluxes.

A comparison of the trapped positron/electron fluxes due to TP and CR sources (Figs. 4 and 9) shows that the TP source produces about 100 times greater positron fluxes than the CR source at the same $L$-shell for positron energies below $100 \mathrm{MeV}$. Beyond energies of $200 \mathrm{MeV}$, the spectral

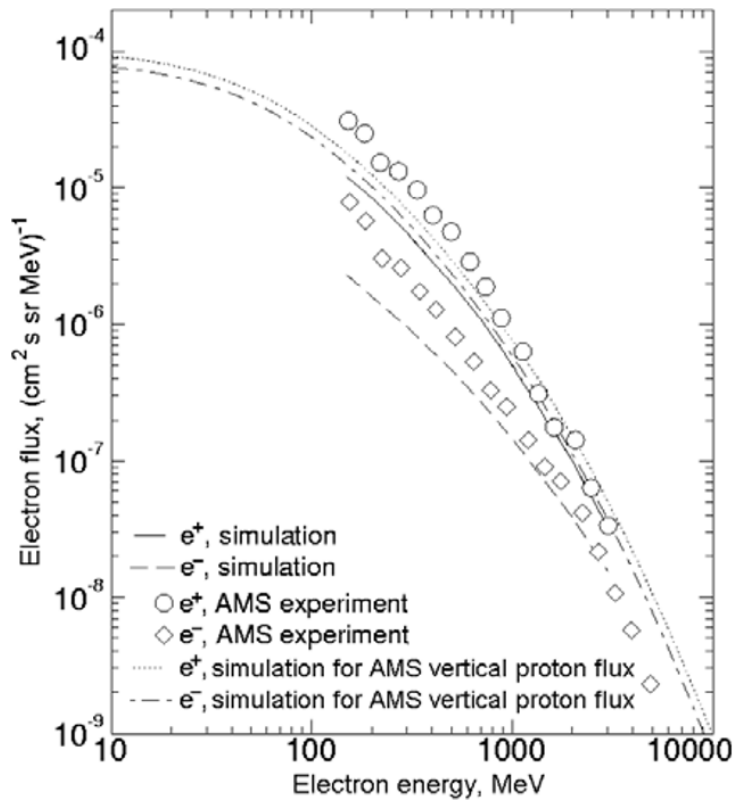

Fig. 9. The results of simulation of trapped positron and electron fluxes born by $\mathrm{CR}$ at $L=1.09$ and comparison with the data of AMS experiment (The AMS collaboration, 2000a). 
distribution decreases sharply and the TP source contributes negligible positron fluxes compared with the CR source at energies above $300 \mathrm{MeV}$.

\section{Discussion}

Our simulation of the positron fluxes agrees with the observed AMS experiment fluxes at energies more than 700 $\mathrm{MeV}$ (Fig. 9). At lower energies there still exist differences that possibly could be related to the next generation particles in the development of nuclear cascade in the atmosphere, which we did not consider in our simulations. The quasitrapped positron and electron fluxes at $L<1.09$ observed in the AMS experiment essentially originated below $100 \mathrm{~km}$ of atmosphere (The AMS collaboration, 2000b) where the next generation secondary particles could be more important than at higher $L$-shells. Only the secondary particles from

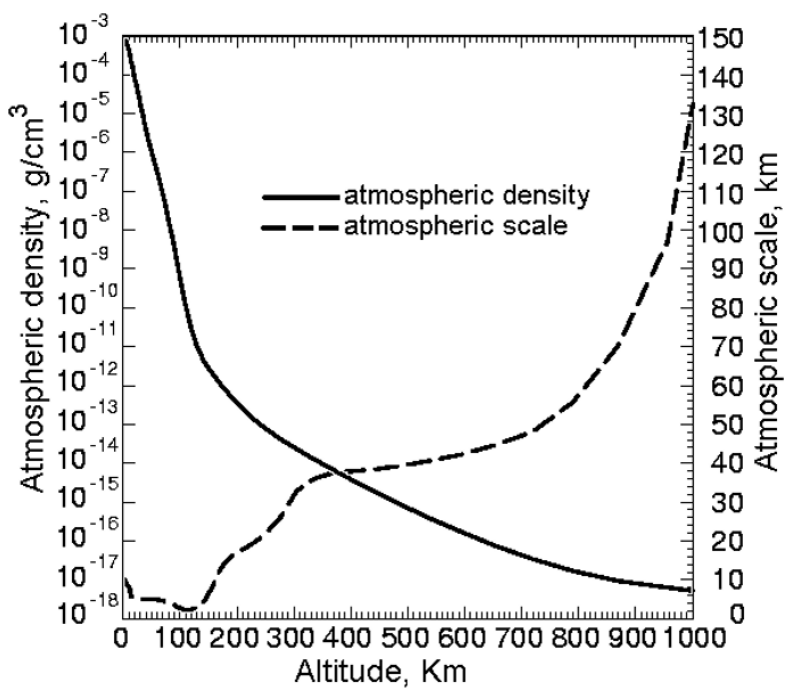

Fig. 10. Atmospheric density and scale according to MSISE model (Hedin, 1991).

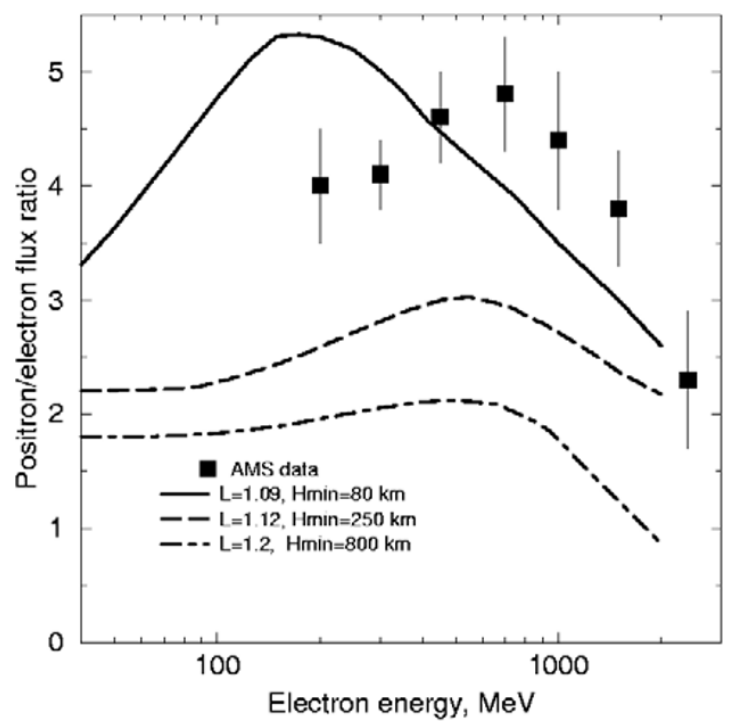

Fig. 11. Positron/electron flux ratio for CR source; the squares are the data of AMS experiment (The AMS collaboration, 2000a). the first interaction of primary protons with the atmospheric atoms were taken into account in our calculations. The probability of a second nuclear interaction with the residual atmospheric atoms is proportional to the square of the atmospheric density and is very small for the atmospheric altitudes considered here $\left(L=1.09\right.$ with $H_{\min }=80 \mathrm{~km} ; 1.12$ with $H_{\min }=250 \mathrm{~km}$; and 1.2 with $H_{\min }=800 \mathrm{~km}$ ).

An essential condition for the existence of positron excess in the fluxes of escaping positrons and electrons is a rapid decrease of the atmosphere density, which scales with altitude. The atmospheric scale height increases with the altitude (see Fig. 10). The positron excess can only be achieved if the atmospheric scale height is less or at least comparable to the Larmor radius of the trapped positron/electron. Thus the positron/electron ratio is dependent on the altitude of the trajectory of the escaping particles and so decreases with the altitude. It is determined mostly by the $H_{\min }$ of a given $L$ shell, which provides a maximal contribution to the integral (Eq. (1)). This is noted in the computed positron/electron flux ratio at $L=1.12$ and $L=1.2$ which are significantly less than that at $L=1.09$ (Fig. 11), essentially due to the increasing atmospheric scale height with the altitude when the $\mathrm{CR}$ azimuthal asymmetry is less pronounced in the fluxes of the trapped secondaries.

\section{Conclusion}

The positron radiation belt flux in the inner magnetosphere of the Earth is simulated considering as the sources the decays of pions produced in nuclear interaction of primary cosmic rays and protons of radiation belt with the residual atmosphere at altitudes above $80 \mathrm{~km}$. The spatial location and the fluxes at the top of magnetic field lines are given for both the sources. In the case of the TP source the positron excess is caused by excess of the positive pions produced in the nuclear interactions. The simulations show that positron radiation belt fluxes from the assumed TP source are limited in spatial distribution at around $L=1.2 \pm 0.1$ with the energy spectrum showing a steep cut-off at energy of about $\approx 300$ $\mathrm{MeV}$. The calculated $e^{+} / e^{-}$flux ratios in the belt due to the TP source are high and attain values of $\geq 7$ in the positron energy range of 10 to $300 \mathrm{MeV}$. In this energy range the simulated fluxes for the CR source of positrons are about 100 times lower than the positron fluxes of the TP origin at altitudes of $L=1.2$. The positrons due to CR origin are also less in the space at $L \geq 1.2$ due to the strong synchrotron energy losses, but their fluxes continue practically equal down to atmospheric boundary, unlike the fluxes due to the TP source which are localized around at $L=1.2$. The estimated spectra due to the CR origin are flatter and measurable fluxes could be obtained up to $2000 \mathrm{MeV}$ energy. The calculations show that the $e^{+} / e^{-}$flux ratio due to the CR source reaches $4-5$ for positrons trapped at the first closed $L$-shells like $L=1.09$. In this case the positron excess is a result of a combination of geophysical effects like an increase of the atmospheric scale height with the altitude, the relatively great positron Larmor gyration radius which is comparable or even greater than the atmospheric scale height and lastly the anisotropic angular distribution of secondary particles. The lower the positron energy the lesser is the $e^{+} / e^{-}$flux ratio produced by the $\mathrm{CR}$ source. The positron/electron ratio 
decreases to $\approx 2$ at $L=1.2$ due to increase of the atmospheric scale.

Acknowledgments. The AP8 model was obtained from the web pages of NASA's National Space Science Data Center. G. Pugacheva and A. Gusev thank the $\mathrm{CNPq}$ for the award of fellowship to work at INPE. The financial support by FAPESP-SP $(93 / 4978-0)$ is acknowledged.

\section{References}

AP8, http://nssdc.gsfc.nasa.gov/space/model/magnetos/radbelt.html, 1996. Barashenkov, V. S. and V. D. Toneev, Interaction of Particles and Atomic Nuclei of High and Superhigh Energies with Nuclei, Atomizdat, Moscow, Russia, 812 pp., 1972.

Basilova, R. N., A. A. Gusev, G. I. Pugacheva, and A. F. Titenkov, High energy trapped electrons in the inner radiation belt, Geom. Aeronom., 22, 671-673, 1982.

Chen, J., T. G. Guzik, Y. Sang, and J. P, Wefel, Energetic helium particles trapped in the magnetosphere, Geophys. Res. Lett., 21, 1583-1586, 1994.

Dementyev, A. V. and N. M. Sobolevsky, SHIELD - universal Monte Carlo hadron transport code: scope and applications, Radiation Measurements, 30, 553-562, 1999.

Freden, S. C. and R. S. White, Particle fluxes in the inner radiation belt, $J$ Geophys. Res., 65(5), 1377-1383, 1960.

Galper, A. M., V. M. Grachev, V. V. Dmitrenko, et al., High energy electrons in the radiation belt of the Earth, 18 Int. Cosmic Ray Conference, Bangalore, India, MG.10-33, 497-500, 1983.

Guinzburg, V. L., Astrophysics of Cosmic Rays, pp. 358, Nauka, Moscow, Russia, 1984 (in Russian).

Gusev, A. A., I. M. Martin, G. I. Pugacheva, A. Turtelli, Jr., and W. N. Spjeldvik, Energetic positron population in the inner zone, Il Nuovo Cimento, Sect. C, 19, 461-465, 1996.

Gusev, A. A., U. B. Jayanthi, G. I. Pugacheva, and W. N. Spjeldvik, Nuclear reactions on rarest atmosphere as a source of magnetospheric positron radiation belt, J. Geophys. Res., 106(A11), 26111-26115, 2001.

Hedin, A. E., Extension of the MSIS thermospheric model into the middle and lower atmosphere, J. Geophys. Res., 96, 1159-1168, 1991.

Machner, H. and J. Hidenbauer, Meson production close to threshold, Journal of Physics G: Nuclear and Particle Physics, 25(10), 231-271, 1999.

Pugacheva, G. I., W. N. Spjeldvik, A. A. Gusev, and I. M. Martin, On the natural energetic positron population in the inner zone of the Earth, $J$. Atmos. Solar-Terr. Phys., 59, 363-369, 1997.

Pugacheva, G. I., W. Gonzalez, A. A. Gusev, U. B. Jayanthi, I. M. Martin, D. Boscher, S. Bourdarier, and W. N. Spjeldvik, Numerical simulation of steady state proton, positron, isotope ion radiation belts and of a sudden creation of transient helium radiation belt during geomagnetic storms, ISEC2001 Radiation Belt Science and Technology, July 23-27, 2001, Queenstown, New Zealand, [http://spacsun.rice.edu/ aac/isec2001/], 2001.

Selesnick, R. S. and R. A. Mewaldt, Atmospheric production of radiation belt light isotopes, J. Geophys. Res., 101(A10), 19745-19759, 1996.

Spjeldvik, W. N., G. I. Pugacheva, A. A. Gusev, I. M. Martin, and N. M. Sobolevsky, Sources of inner Radiation Zone Energetic Helium Ions: cross-field transport versus in-situ nuclear reactions, Adv. Space Res., 21, 1675-1678, 1998a.

Spjeldvik, W. N., G. I. Pugacheva, A. A. Gusev, I. M. Martin, and N. M. Sobolevsky, Hydrogen and helium isotope inner radiation belts in the Earth's magnetosphere, Annales Geophysicae, 16, 931-939, 1998b.

The AMS collaboration, Protons in near Earth orbit, Phys. Lett. B, $\mathbf{4 7 2}$, 215-226, 2000a.

The AMS collaboration, Leptons in near Earth's orbit, Phys. Lett. B, 484, $10-17,2000 \mathrm{~b}$

Voronov, S. A., A. M. Galper, V. G. Kirillov-Ugryumov, S. V. Koldashev, and A. V. Popov, High energy electrons and positrons in the Earth's radiation belt, Geom. and Aeronom., 27(3), 424-427, 1987.

A. A. Gusev (e-mail: cshorng@earth.sinica.edu.tw), U. B. Jayanthi, G. I. Pugacheva, V. M. Pankov, and N. Schuch 